\title{
Effects of Anthropogenic Climate Change on the Occurrence of Supercellular Tornadoes in the USA
}

Samuel Inskip, Monash University

\section{Abstract}

The United States of America (USA) experiences the most tornadoes of any nation in the world, with these extreme weather events causing many deaths and billions of dollars of damage each year. With the recent warming of the climate due to human activity, it is likely that the occurrence of USA tornadoes will be affected. However, exactly how the spatial and temporal distribution of tornadoes in the USA will be affected by climate change is still an area of active research. The trends in the literature show that as the climate has warmed, there has been an observed clustering of USA tornadoes onto fewer, more active tornado days, as well as an eastward movement of the average centre of USA tornado activity. This review shows that these observed changes could be explained by shifts in environmental parameters related to tornado formation due to anthropogenic climate change. This research will allow for better tornado preparedness and prediction in tornado-affected areas as the climate warms. Future research may focus on more precisely modelling the impacts of global warming on USA tornado occurrence by using higher-resolution climate models.

Keywords: USA tornado climatology; temporal clustering of USA tornadoes; spatial redistribution of USA tornadoes; Tornado Alley; supercell formation

\section{Introduction}

A tornado is a violently rotating column of air, originating from a thunderstorm, that makes contact with the ground (Guo et al., 2016), with supercellular tornadoes being the most powerful and destructive type. This type of tornado originates from a special type of thunderstorm, known as a supercell, that is characterised by a strong, rotating column of air in the middle of the storm, called a mesocyclone (Glickman, 2000).

The USA is particularly vulnerable to extremely strong and destructive tornadoes due to its geographical location being ideal for the formation of powerfully rotating supercells. The flat 
central plains of the USA allow for warm, moist air from the Gulf of Mexico to be overlain by cooler, dryer continental air from higher elevations to the west, causing temperatures to decrease rapidly with height (Schultz et al., 2014). This strong vertical temperature gradient results in a high convective available potential energy (CAPE), which is a measure of how likely air is to rise and form a thunderstorm, with higher CAPE values generally resulting in stronger storms (Brooks et al., 2014). Additionally, at the mid-latitudes where the USA is located, there is a large change in wind speed with altitude called 'wind shear', which is essential for generating the rotating mesocyclone that characterises supercells. This combination of high CAPE and high wind shear in the Great Plains of the central USA makes the area well suited to generating powerful tornadoes. Strong tornadoes also occur in the south-eastern USA, outside of the Great Plains, where they tend to form from high wind shear, low-CAPE supercells; these remain poorly understood in the literature (Wade and Parker, 2021).

The USA experiences the most tornadoes of any nation in the world (Guo et al., 2016), with tornadoes resulting in an average of 110 deaths and billions of dollars of damage each year (Tippett and Cohen, 2016). Moreover, anthropogenic climate change has been found to be affecting key factors in tornado and supercell formation, such as wind shear and CAPE (Diffenbaugh et al., 2013). Due to the destructive nature of tornadoes, it is imperative that we understand if and how tornado formation in the USA will be affected by climate change in order to mitigate the risk posed to lives and infrastructure by these extreme weather events.

Therefore, the aim of this literature review is to understand how anthropogenic climate change may be affecting the formation patterns of tornadoes within the contiguous USA. It seeks to consolidate the findings of recent research about the changes in the temporal and spatial distribution of tornado formation in the USA since the 1950s and uncover the overarching trends in recent USA tornado climatology. It will also analyse recent research to uncover possible mechanisms for how anthropogenic climate change could be causing the observed changes in the tornado record.

\section{Changes in the frequency of USA tornadoes}

In the USA, the National Weather Service's Storm Prediction Centre maintains a database that contains information about the location and time of every recorded tornado in the USA that has occurred since 1954 (Schaefer and Edwards, 1999). This database has been used to analyse 
the annual count of tornado days in the USA, which are defined as any day when at least one tornado has formed in the entire USA. This is essentially a measure of the number of days per year when there are suitable conditions for tornado formation to occur. It has been found that, since 1954, there has been a significant downwards trend in the annual number of tornado days in the USA (Brooks et al., 2014; Elsner et al., 2015; Moore, 2017a; Moore, 2017b). In particular, Brooks et al. (2014) found that the average annual number of tornado days has decreased by 33 per cent, from 150 in the 1950 s to 100 in the 2010s.

This trend implies that the number of days in the USA with conditions suitable for tornadogenesis (i.e. tornado formation) has decreased over the past half-century, possibly in response to anthropogenic global warming. This potential link is supported by the fact that the decreasing trend in tornado days began in the early 1980s (Elsner et al., 2015), which corresponds with a period of increasing average temperatures in the USA from 1984 onwards (Agee et al., 2016) (Figure 1).

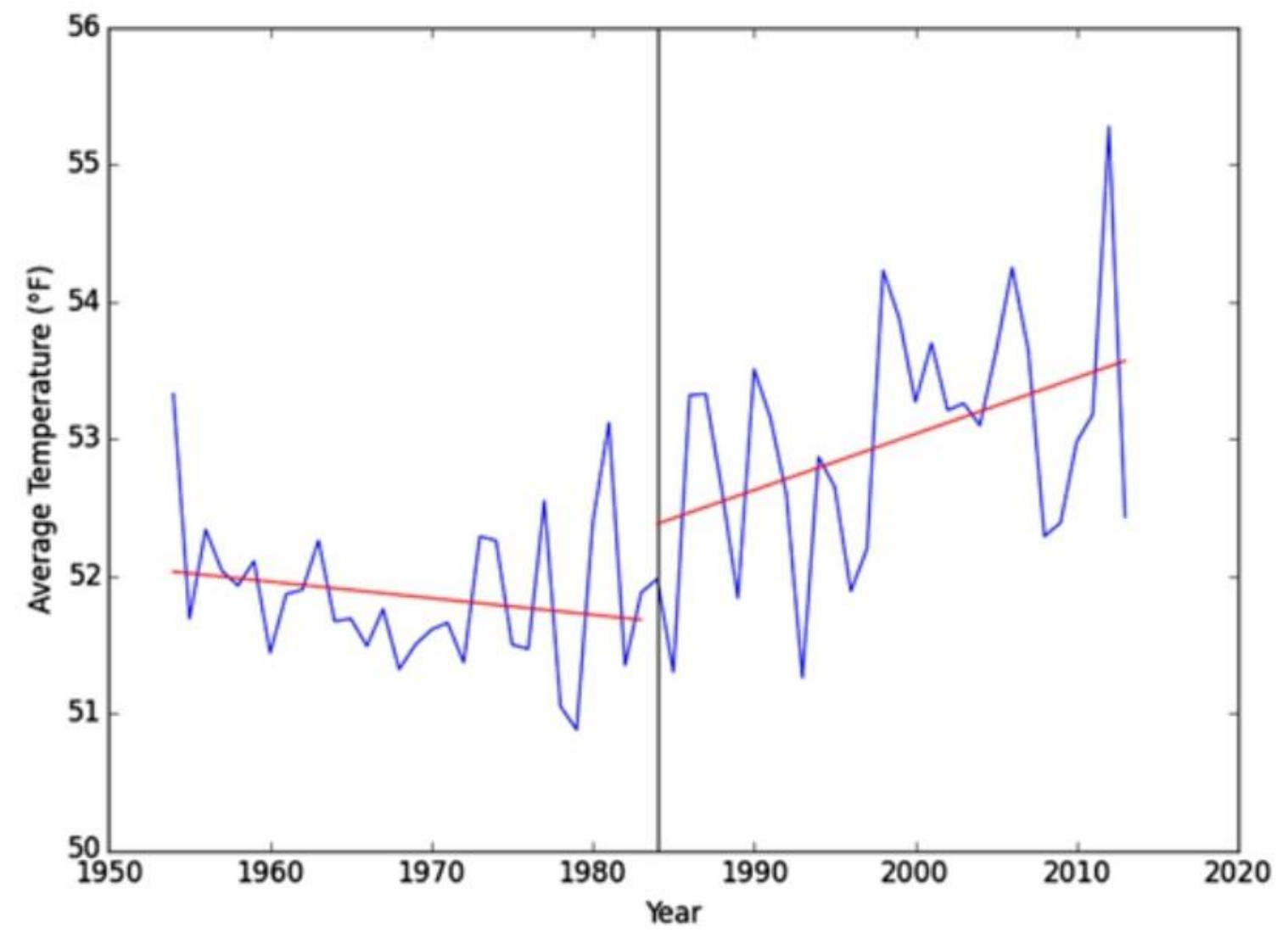


Figure 1: Average annual temperatures in the continental USA from 1954 to 2013 split into a 'cold' period (1954-1983) and a 'warm' period (1984-2013) (Agee et al., 2016). (C) American Meteorological Society. Used with permission.

Furthermore, it can be seen that the average number of tornadoes per tornado day has noticeably increased since 1974 (Brooks et al., 2014; Elsner et al., 2015; Moore, 2017a; Moore, 2017b). Moore (2017a) found that this overall increase was primarily due to a statistically significant increase in the number of tornado days with 30+ tornadoes, with a corresponding decrease in tornado days with 1-10 tornadoes per day in the USA from 1974 to 2014. These concurrent trends have caused the mean number of tornadoes per tornado day to have increased from 3.75 from 1975 to 1980 to 5.46 from 2010 to 2014 (Moore, 2017a).

These findings indicate that, since the 1970s, tornadoes in the USA have been tending to cluster together on fewer tornado days, with each tornado day producing more tornadoes on average. This implies that as the climate in the USA has been warming due to anthropogenic climate change, the total number of days with favourable weather conditions for tornadoes has decreased. However, days when suitable conditions do occur have tended to produce larger and more severe tornado outbreaks.

\section{Changes in the spatial distribution of tornadoes in the USA}

Traditionally, the centre of tornado formation in the USA has been centred on the Great Plains in the central USA in an area known as Tornado Alley (Schultz et al., 2014), which contains parts of South Dakota, Nebraska, Oklahoma, Kansas and Texas (Doswell et al., 2012).

To investigate how a warming climate may be affecting this distribution of tornadoes, Agee et al. (2016) split the modern tornado record into a 'cold' period (1954-1983) and a 'warm' period (1984-2013) based on the average temperature trends of the USA during these periods (Figure 1). By dividing the eastern USA into $2.5^{\circ} \mathrm{x} 2.5^{\circ}$ grid boxes and comparing the tornado count in each grid box across both periods, the authors found that the area of maximum tornado activity in the USA has shifted eastwards from the traditional tornado centre of Oklahoma during the 'cold' period to Mississippi, Alabama, Louisiana and Tennessee in the southeastern USA during the 'warm' period (Figure 2). 


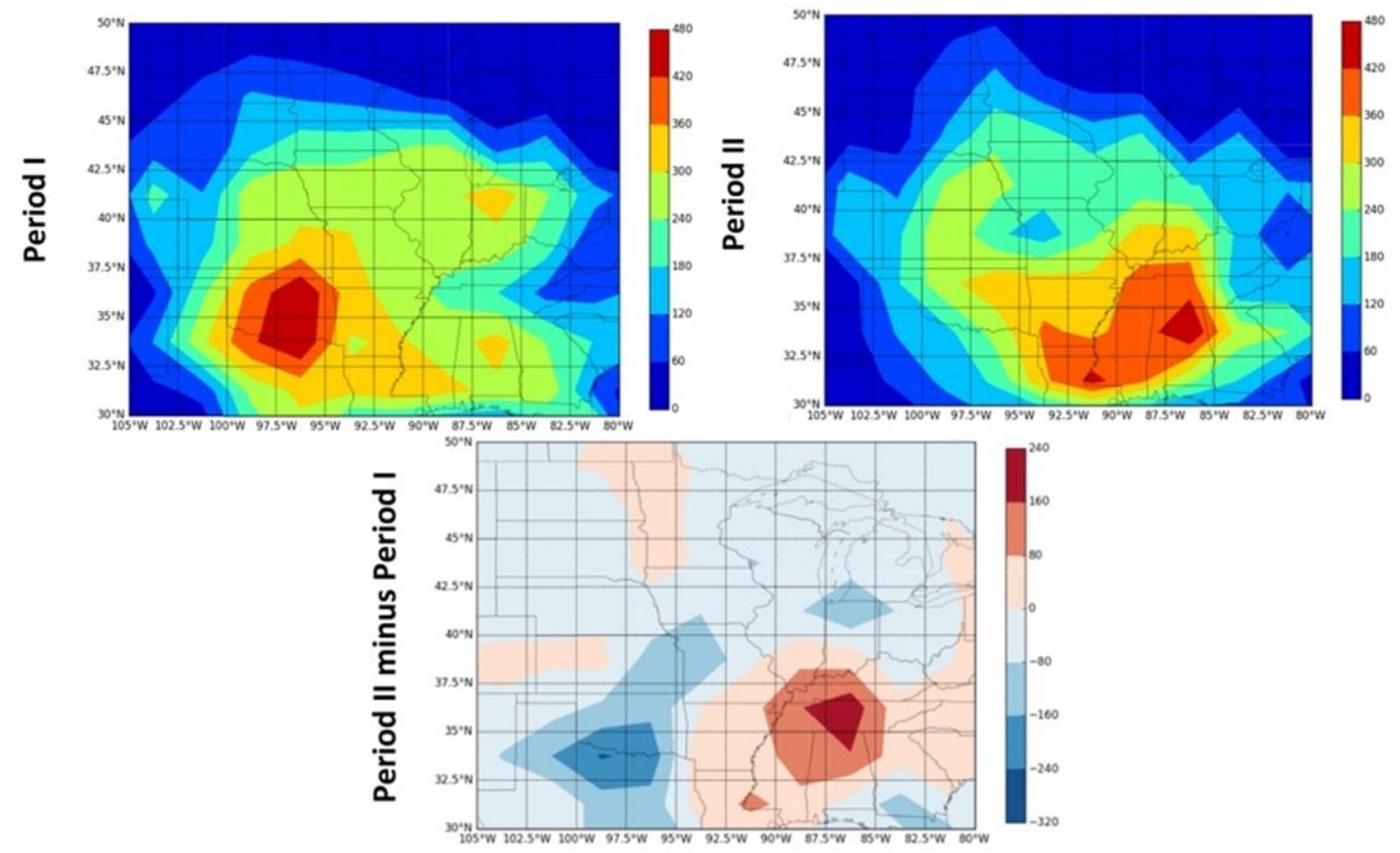

Figure 2: Map of tornado counts for the eastern USA for Period I (1954-1983), Period II (1984-2013) and Period II minus Period I (modified from Agee et al., 2016). @ American Meteorological Society. Used with permission.

Similar results have been obtained by Gensini and Brooks (2018) by calculating the annual maximum significant tornado parameter (STP) in $1^{\circ} \times 1^{\circ}$ boxes across the eastern USA and calculating how much it has changed for each box from 1979 to 2017. The STP is a predictor of how likely a tornado is to form in an area based on various environmental conditions such as wind shear, CAPE and storm-relative helicity (SRH; i.e. the strength of a supercell's mesocyclone). A statistically significant decrease in STP was found in Tornado Alley, with a corresponding increase in STP to the east around Mississippi, Louisiana, Alabama, Tennessee, Arkansas, Kentucky, Missouri, Illinois and Indiana.

These findings imply that, since 1979, the area where tornado-favourable conditions occur in the USA has shifted to the east, away from Tornado Alley, supporting the observed eastward shift in tornado reports found by Agee et al. (2016) and Moore (2017a). Moreover, the period 1979-2017 corresponds with the period of warming temperatures in the USA identified by Agee et al. (2016) from 1984 to 2013, which implies that anthropogenic global warming may be a factor in this shift in tornado activity. 
Moore (2017a) has proposed that this eastward movement may be due to the significant increase in days with 50+ tornadoes in the USA since 1974, which are more likely to occur to the east of Tornado Alley. These larger tornado outbreaks occurring to the east of Tornado Alley may be related to the increase in annual maximum STP in the east, identified by Gensini and Brooks (2018), that would make tornado outbreaks more likely to occur in this region.

\section{Challenges in obtaining accurate tornado records for analysis}

It has been suggested by some researchers that some or all of these trends in tornado climatology could be explained by changes in tornado reporting practices, rather than changes in environmental conditions (Kunkel et al., 2013; Brooks et al., 2014).

These ideas may have some credence, as moderately detailed and accurate tornado records in the USA have only been available since the 1950s. Moreover, the introduction of Doppler radar in the 1990s, which can detect tornadoes remotely, caused a large artificial increase in tornado counts as a large number of weak tornadoes began to be detected that previously went unnoticed (Potvin et al., 2018). Even with this improved technology, Potvin and colleagues estimate that only 45 per cent of tornadoes in the USA from 1975 to 2016 have been reported due to the large number of tornadoes occurring in rural areas.

Because of this short period of accurate tornado data, the incompleteness of the data and the large change in tornado reporting methods with the introduction of Doppler radar, it is difficult to determine whether the aforementioned trends in tornado climatology have been caused by climate change or changes in tornado reporting.

To try and minimise the impact of these changes in reporting, most literature on USA tornado climatology has only considered tornadoes that are ranked stronger than (E)F1 on the (enhanced) Fujita scale in their analysis. This scale is a measure of tornado power based on the measured wind speed and damage done by the tornado, with (E)F0 tornadoes being the weakest and (E)F5 tornadoes being the strongest (Marshall et al., 2004). Since the 1950s, (E)F0 tornado reports have increased by 14-16 reports per year, while tornadoes rated stronger than

(E)F1 have seen no significant increase over the same period (Tippett 2014) (Figure 3). 


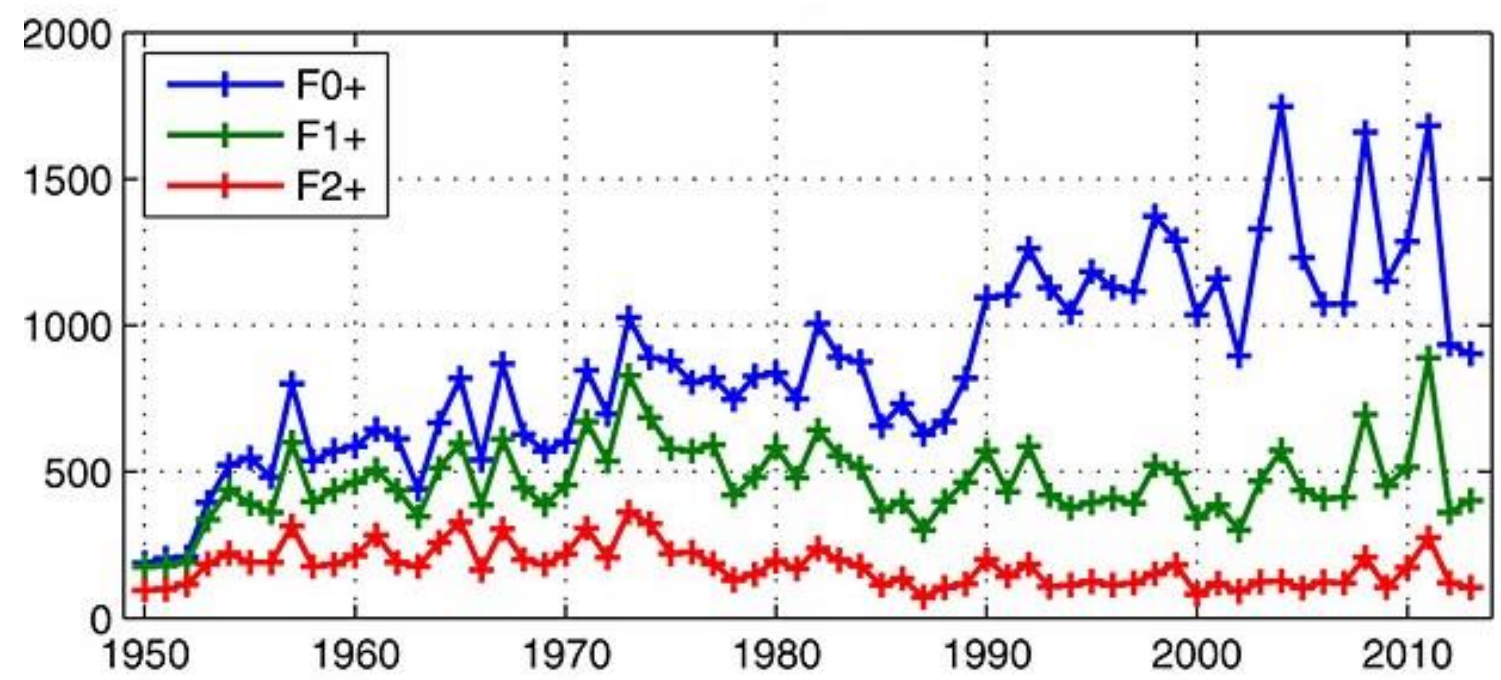

Figure 3: Annual USA tornado count for all tornadoes (blue), F1+ tornadoes (green) and F2+ tornadoes. Modified from Tippett (2014).

However, as the majority of tornadoes are rated as (E)F0s, excluding them from the data is not an ideal solution, as it biases the models towards trends in the formation of stronger tornadoes, which may not be representative of overall USA tornado climatology.

To try and overcome these limitations in reported tornado data, Tippett et al. (2012; 2014) developed a model called the tornado environment index (TEI) that predicts the number of tornadoes in a given timeframe based on average monthly rainfall from thunderstorms and average monthly SRH, which have both been more accurately recorded than annual tornado counts. Applying the TEI to USA climate data from 1979 to 2013, Tippett (2014) found that it accurately modelled changes in tornado activity from year to year, including the observed increase in USA tornado variability (Brooks et al., 2014; Elsner et al., 2015; Guo et al., 2016; Tippett and Cohen, 2016), as seen in Figure 4. 


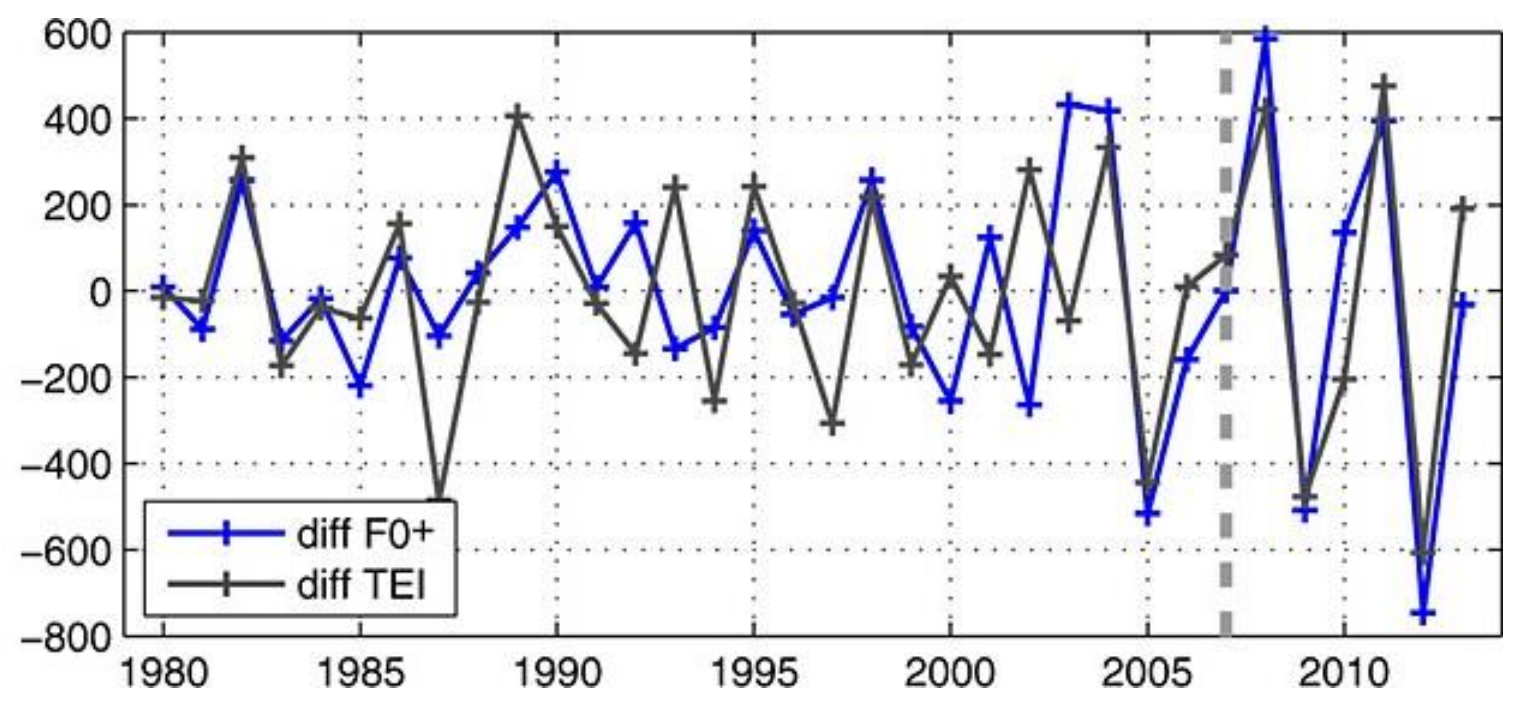

Figure 4: Year-to-year differences in the reported number of tornadoes in the USA (blue) and the predicted difference using the TEI (black). Modified from Tippett (2014).

As the TEI is based on accurately measured environmental parameters - rather than the problematic tornado record - this supports the idea that the observed changes in tornado reports are due to changes in the climate rather than changes in recording practices.

\section{Mechanisms for how a warming climate is affecting USA tornado frequency}

Due to our current incomplete understanding of how exactly tornadoes form during supercell events, it is very difficult to model how anthropogenic climate change will affect tornado formation directly. However, a good indication of how USA tornado climatology will change can be found by modelling how factors such as wind shear and CAPE -known to affect tornadogenesis - will change in a warming climate.

The current consensus in the literature is that a warming climate will cause an increase in days in the USA with favourable combinations of CAPE and wind shear for producing severe storms, and possibly tornadoes (Diffenbaugh et al., 2013; Gensini and Mote, 2015; Seeley and Romps, 2015; Tippett et al., 2015). This would suggest that the annual number of tornado days in the USA should be increasing with a warming climate instead of the observed decrease. However, CAPE and wind shear are not the only variables affecting storm formation, as convective inhibition (CIN) - which is how difficult it is for air to rise and form a storm - has also been predicted to increase with a warming climate (Tippett et al., 2016). Higher CIN values mean 
that it is harder for convection to initiate, so fewer storms form in a higher CIN environment, which could be counteracting the predicted increase in thunderstorm activity.

This idea is supported by the findings of Trapp and Hoogewind (2016), who simulated three historical tornado outbreaks in a climate model with climate conditions from the 1990s, and with predicted climate conditions in the 2090s in a business-as-usual global warming model. It was discovered that, compared to the 1990s simulation, many supercells in the 2090s simulation failed to initiate due to the increased CIN in a warmer climate. However, when supercells did manage to overcome the increased CIN, they were found to have stronger vertical updrafts and stronger rotation due to the increased CAPE in the 2090s, which is conducive to the formation of larger tornado outbreaks. This implies that in a warmer climate, the number of supercells that form will decrease overall due to increased CIN; however, when they do form, they will be more powerful and more likely to produce multiple tornadoes due to the increased CAPE.

This predicted trend in storm frequencies is notably similar to the observed trend in USA tornado frequencies, where the total number of tornado days has decreased, while the number of tornadoes per tornado day has increased (Brooks et al., 2014; Elsner et al., 2015; Moore, 2017a; Moore, 2017b). As a result, it is likely that increasing values of CAPE and CIN in a warming climate are the climatological mechanism behind the observed changes in USA tornado frequency.

\section{Mechanisms for the observed change in USA spatial tornado distribution}

Multiple studies have used climate models to map how the distribution of CAPE and wind shear will change across the USA due to anthropogenic climate change (Diffenbaugh et al., 2013; Gensini and Mote, 2015; Seeley and Romps, 2015). It was found by Gensini and Mote (2015) that in the 1990s, the area with the highest frequency of days with favourable CAPE and wind shear for supercell formation in the USA during storm season (spring) was eastern Oklahoma, western Arkansas and south-western Missouri, which corresponds well with the area of maximum tornado activity in the period 1954-1983 identified by Agee et al. (2016). In the 2080s, this area of favourable conditions is predicted to expand eastwards to cover all of Arkansas and Louisiana, as well as parts of Texas, Mississippi and Missouri. 
The eastward expansion of this region of favourable supercell conditions in a warming climate is predicted to cause a 27 per cent increase in future severe weather events, such as tornadoes, in the Mississippi, Ohio and Tennessee River valleys (Gensini and Mote, 2015), which lie to the east of Tornado Alley. This correlates well with the observed eastwards shift of the centre of USA tornado formation (Agee et al., 2016; Moore, 2017a), and suggests that an expansion of areas with favourable CAPE and wind shear conditions in the USA due to anthropogenic climate change is likely the mechanism behind the observed eastwards shift in USA tornado activity.

\section{Conclusion}

In conclusion, anthropogenic climate change is most likely affecting the occurrence of USA tornadoes in two main ways. Firstly, it is causing USA tornadoes to cluster together into less frequent but more active tornado days. A possible mechanism for this is that a warming climate increases CAPE, which increases supercell severity, as well as CIN, which decreases supercell frequency. This would cause supercells to form less often, but to be more severe and thus produce larger tornado outbreaks - when they do occur.

Secondly, climate change is causing the mean centre of tornado activity in the USA to move eastwards, away from Tornado Alley. This is likely due to global warming causing an eastwards expansion of the area where favourable CAPE and wind shear conditions for supercell formation occur in the USA. As a result, more severe supercells, and therefore tornadoes, are forming to the east of Tornado Alley, causing the mean centre of tornado activity to move east.

As the proposed climatological mechanisms behind these trends in tornado climatology are predicted to become more pronounced with future climate change, it is likely that the observed changes in USA tornado climatology will continue for the foreseeable future as average global temperatures increase.

The trends identified in this report are a good starting point in our understanding of the relationship between USA tornadoes and anthropogenic climate change. However, due to the aforementioned limitations of the tornado database, our limited understanding of tornadogenesis, and the limited resolution of climate models, it is impossible to definitively say whether these observed changes in USA tornado climatology are being caused by anthropogenic climate change, or are due to a longer-term natural climate variability. To 
Reinvention: an International Journal of Undergraduate Research 14:2 (2021)

obtain a definitive answer, more research needs to be done in this area using higher-resolution climate modelling and a better understanding of tornadogenesis. 


\section{List of figures}

Figure 1: Average annual temperatures in the continental USA from 1954 to 2013 split into a 'cold' period (1954-1983) and a 'warm’ period (1984-2013) (Agee et al., 2016). ( $)$ American Meteorological Society. Used with permission.

Figure 2: Map of tornado counts for the eastern USA for Period I (1954-1983), Period II (19842013) and Period II minus Period I (modified from Agee et al., 2016). (c American Meteorological Society. Used with permission.

Figure 3: Annual USA tornado count for all tornadoes (blue), F1+ tornadoes (green) and F2+ tornadoes. Modified from Tippett (2014).

Figure 4: Year-to-year differences in the reported number of tornadoes in the USA (blue) and the predicted difference using the TEI (black). Modified from Tippett (2014).

\section{References}

Agee, E., J. Larson, S. Childs and A. Marmo (2016), 'Spatial redistribution of U.S. tornado activity between 1954 and 2013', Journal of Applied Meteorology and Climatology, 55, 1681-97

Brooks, H. E., G.W. Carbin and P. T. March (2014), 'Increased variability of tornado occurrence in the United States', Science, 346, 349-52

Diffenbaugh, N. S., M. Scherer and R. J. Trapp (2013), 'Robust increases in severe thunderstorm environments in response to greenhouse forcing', Proceedings of the National Academy of Sciences of the United States of America, 110, 16361-66

Doswell, C. A., G. W. Carbin, and H. E. Brooks (2012), 'The tornadoes of spring 2011 in the USA: A historical perspective', Weather, 67, 88-94

Elsner, J. B., S. C. Elsner and T. H. Jagger (2015), 'The increasing efficiency of tornado days in the United States', Climate Dynamics, 45, 651-59 
Gensini V. A. and H. E. Brooks (2018), 'Spatial trends in United States tornado frequency', Climate and Atmospheric Science, 1

Gensini, V. A. and T. L. Mote (2015), 'Downscaled estimates of late 21st century severe weather from CCCSM3', Climactic Change, 129, 307-21

Glickman, T. S. (2000), Glossary of Meteorology, Boston, MA: American Meteorological Society

Guo, L., K. Wang and H. B. Bluestein (2016), 'Variability of tornado occurrence over the continental United States since 1950', Journal of Geophysical Research: Atmospheres, 121, 6943-53

Kunkel, K. E., T. R. Karl, H. Brooks, J. Kossin, J. H. Lawrimore, D. Arndt, L. Bosart, D. Changnon, S. L. Cutter, N. Doesken, et al. (2013), 'Monitoring and understanding trends in extreme storms: State of knowledge', Bulletin of the American Meteorological Society, 94, $499-514$

Marshall, T. P., J. S. McDonald and G. S. Forbes (2004), 'The enhanced Fujita (EF) scale', 22nd Conference on Severe Local Storms, Carrollton, 2004

Moore, T. W. (2017a), 'On the temporal and spatial characteristics of tornado days in the United States', Atmospheric Research, 184, 56-65

Moore, T. W. (2017b), 'Annual and seasonal tornado trends in the contiguous United States and its regions', International Journal of Climatology, 38, 1582-94

Potvin, C. K., C. Broyles, P. S. Skinner, H. E. Brooks and E. Rasmussen (2018), 'A Bayesian hierarchical modeling framework for correcting reporting bias in the U.S. tornado database', Weather and Forecasting, 34, 15-30

Schaefer, J. T. and R. Edwards (1999), 'The SPC tornado/severe thunderstorm database', in 11th Conference on Applied Climatology, 1999, Dallas, Texas: American Meteorological Society, pp. 215-20

Seeley, J. T. and D. M. Romps (2015), 'The effect of global warming on severe thunderstorms in the United States', Journal of Climate, 28, 2443-58 
Schultz, D. M., Y. P. Richardson, P. M. Markowski and C. A. Doswell (2014), 'Tornadoes in the Central United States and the "clash of air masses", Bulletin of the American Meteorological Society, 94, 1704-12

Tippett, M. K. (2014), 'Changing volatility of U.S. annual tornado reports', Geophysical Research Letters, 41, 6956-61

Tippett, M. K. and J. E. Cohen (2016), 'Tornado outbreak variability follows Taylor's power law of fluctuation scaling and increases dramatically with severity', Nature Communications, 7 (1), 10668

Tippett, M. K., A. H. Sobel and S. J. Camargo (2012), 'Association of U.S. tornado occurrence with monthly environmental parameters', Geophysical Research Letters, 39 (2), 1-6

Tippett, M. K., A. H. Sobel, S. J. Camargo and J. T. Allen (2014), 'An empirical relation between U.S. tornado activity and monthly environmental parameters', Journal of Climate, 27, 2983-99

Tippett, M. K., J. T. Allen, G. A Vittorio and H. E. Brooks (2015), 'Climate and hazardous convective weather', Current Climate Change Report, 1, 60-73

Tippett, M. K., C. Lepore and J. E. Cohen (2016), 'More tornadoes in the most extreme U.S. tornado outbreaks', Science, 354, 1419-23

Trapp, R. J. and K. A. Hoogewind (2016), 'The realization of extreme tornadic storm events under future anthropogenic climate change', Journal of Climate, 29, 5251-65

Wade, A. R. and Parker, M. D. (2021). 'Dynamics of simulated high-shear, low-CAPE supercells'. Journal of the Atmospheric Sciences, 78(5), 1389-410.

\section{Glossary}

Anthropogenic: Something caused by human activity.

Convective available potential energy (CAPE): The amount of work done by the buoyant force on a parcel of air rising through the atmosphere. Positive CAPE will cause the air parcel to rise, and vice versa. 
Convective inhibition (CIN): The amount of energy required by an air parcel to overcome its negative buoyancy and rise away from the ground.

Doppler radar: A type of radar that uses the Doppler Effect to measure the velocity of objects. It is commonly used to measure wind speeds and precipitation in thunderstorms.

Enhanced Fujita Scale: An updated version of the Fujita scale, introduced in the USA in 2007.

Fujita scale: A scale for rating the strength of a tornado based on measured wind speed and observed damage done by the tornado. The weakest tornadoes are F0s; the strongest are F5s.

Mesocyclone: A strong, rotating updraft in a supercell.

Significant tornado parameter (STP): A predictor of how likely a tornado is to form in a given area based on environmental conditions including wind shear, CAPE and SRH.

Storm-relative helicity (SRH): A measure of the potential for the formation of a rotating updraft (i.e. a mesocyclone) in a thunderstorm.

Supercell: A type of thunderstorm characterised by a strong, rotating column of air in the middle of the storm known as a mesocyclone.

Tornado: A violently rotating column of air, originating from a thunderstorm, that makes contact with the ground.

Tornado Alley: An area in the Great Plains of the USA that experiences the most frequent tornadoes in the USA.

Tornado day: Any day when at least one tornado forms somewhere in a given geographical area (e.g., the contiguous USA).

Tornado environment index (TEI): A function of SRH and monthly rainfall from thunderstorms that predicts the number of tornadoes that will form in a given timeframe.

Tornadogenesis: The process by which a tornado is formed.

Tornado outbreak: The occurrence of multiple tornadoes spawned from the same weather system. Generally, at least six to ten tornadoes must form for it to be classified as an outbreak. 
Updraft: A current of rising air within a thunderstorm.

Wind shear: Change in wind speed over a given distance in the atmosphere.

To cite this paper please use the following details: Inskip, S. (2021), 'Effects of Anthropogenic Climate Change on the Occurrence of Supercellular Tornadoes in the USA', Reinvention: an International Journal of Undergraduate Research, Volume 14, Issue 2, https://reinventionjournal.org/article/view/716. Date accessed [insert date]. If you cite this article or use it in any teaching or other related activities please let us know by e-mailing us at Reinventionjournal@warwick.ac.uk 